\title{
Frequency of methicillin-resistant Staphylococcus aureus (MRSA) in nose and cellular phone of medical and non-medical personnel of emergency departments of Ghaem hospital in Mashhad city
}

\author{
Hadi Safdari $^{\mathrm{a}, \mathrm{b}, *}$, Ehsan Aryan $^{\mathrm{a}}$, Hamid Sadeghian ${ }^{\mathrm{b}}$, Seyyedeh Fatemeh Shams ${ }^{\mathrm{a}}$, Mahdi Aganj ${ }^{\mathrm{a}}$ \\ a Antimicrobial Resistance Research Center, Mashhad University of Medical Sciences, Mashhad, Iran \\ ${ }^{\mathrm{b}}$ Department of Laboratory Sciences, School of Paramedical Sciences, Mashhad University of Medical Sciences, Mashhad, Iran
}

\section{A R T I C L E I N F O}

\section{Keywords:}

Staphylococcus aureus

MRSA

Antimicrobial resistance

Contamination

Cell phone

Susceptibility test

\begin{abstract}
A B S T R A C T
Background: Staphylococcus aureus is one of the commensal bacteria and components of nose normal flora in $30-40 \%$ of people. The cellular phone is infected through the hands of a person who has already been infected with nasal discharge, in the hospital. Patients and family members with a weakened immune system are at high risk of contamination; the treatment procedure is difficult.

Materials and methods: 200 individuals who were working in the emergency departments of Ghaem were included in the study. Sampling was done by three sterile swabs; two swabs from the nose and one from the surface of the cellular phone. The diagnosis was done according to the staphylococcus biochemical tests. Beta-lactamase positive staphylococcus was identified by nitrocefin. MIC method was performed using E-test strips and agar dilution for detecting the MRSA.

Results: 86 out of 200 nasal, and 200 cellular phone samples were diagnosed as beta-lactamase positive staphylococci and sensitivity test was done. 17 out of 86 beta-lactamase positive Staphylococcus aureus samples were resistant to cefoxitin, piperacillin, ceftriaxone, and cefotaxime; just one of these 17 samples was resistant to vancomycin. 24 samples were contaminated with healthcare-associated MRSA (HA-MRSA). Totally, cellular phone and nasal discharge of $12 \%$ of studied individuals were infected with the HA-MRSA.

Conclusion: Cell phone usage should be prohibited in the hospital sections. Disinfection of the mobile phone should be taken when leaving the hospital, along with hand, face, and nose washing seriously. It is suggested to evaluate all persons working in hospitals regarding the contamination to MRSA.
\end{abstract}

\section{Introduction}

Staphylococcus aureus is a member of the micrococci family which is coagulase-positive; it is positive in gram staining too. Staphylococcus aureus is the main cause of septicemia, endocarditis, osteomyelitis, pneumonia, toxic shock syndrome, food poisoning, carbuncle, and furuncle. These complications are more prevalent among patients with a weakened immune system. It is found in the anterior part of the nose in adults and pediatrics as the primary source; it is found in the nose of $20-40 \%$ of healthy individuals too. The number of bacteria is higher in people who work in the hospital sectors. Staphylococcus aureus carriers are the main cause of Staphylococcal infections. ${ }^{1,2}$ Staphylococci were sensitive to penicillin before 1940. It was the first antibiotic that was used for treatment in 1941. After a short time, strains containing betalactamase enzymes were generated in Staphylococcus aureus and resistance was spread in the environment. The lack of binding of $\beta$ - lactams to Penicillin-Binding proteins (PBPs) is the main cause of Staphylococcus aureus resistance to the antibiotics. These proteins are responsible for building the bacterial cell wall; they are targeted by betalactam antibiotics. PBP2a is one of the subgroups of mutant PBPs; it is $78 \mathrm{kDa}$. It increases the resistance of Staphylococcus aureus to betalactams due to the high expression and low binding to beta-lactams. ${ }^{3,4}$

The production of this new protein is linked to the mec genes which are found in the bacterium genome. The emergence of MRSA strains is a therapeutic problem in patients. PBP2a is encoded by the mecA gene and transported by the SCCmec (Staphylococcal Cassette Chromosome mec) large cassette gene. This cassette consists of three parts: ccr complex, mec complex, and $\mathrm{J}$ regions. There are five main types of SCCmec (types VI-I), based on the characteristics of this genetic region. Mainly, it causes resistance to methicillin and other beta-lactams. The presence or the absence of the mecA gene determine methicillin resistance or susceptibility. MRSA is resistant to beta-lactam antibiotics

\footnotetext{
* Corresponding author. Antimicrobial Resistance Research Center, Mashhad University of Medical Sciences, Mashhad, Iran.

E-mail address: hadiSafdari43@gmail.com (H. Safdari).
} 
such as penicillin, methicillin, nafcillin, and oxacillin. MRSA is one of the most prevalent pathogens in the world that is responsible for a wide range of nosocomial infections. ${ }^{5,6}$ Studies have shown that contaminated medical staff (including doctors, nurses, and even service personnel in departments) are common sources of antibiotic-resistant Staphylococcus aureus epidemics in the hospital. MRSA which are separated from hospitals are called Healthcare-associated MRSA (HAMRSA), and those which are separated from the community are named Community-Acquired MRSA (CA-MRSA). When you use a mobile phone, nose colonized staphylococci are placed on the mobile surface. According to the drought resistance of bacterium, it will be able to survive on the mobile surface. Mobile reuse will contaminate the fingers and then other consumables, and eventually will transfer to the next person. Hospital staff including physicians, nurses, health care providers, and even service personnel, are at risk. ${ }^{7-9}$

Today, despite therapeutic antibiotics, MRSA strains have spread in many parts of the world, especially in hospitals and health centers. ${ }^{10}$ Therefore, we aimed to obtain samples from nasal discharge and cell phones in the emergency Departments of Ghaem Hospital in Mashhad city, Iran. The isolates of MRSA-HA were examined for antibiotic resistance.

\section{Material and methods}

In the present study, 200 medical personnel of Ghaem hospital of Mashhad were selected from five emergency departments including internal, neurosurgery, surgery, pediatrics and heart. Sampling was done during May-November 2017.146 out of studied individuals were female $(85.6 \%)$, and $17(14.2 \%)$ were male. Sampling was done by three swabs from each of the subjects. Samples were taken from each nostril using two sterile wet swabs. The swab was passed about one $\mathrm{cm}$ in the nostril and rotated; the rotation was done five times. The third swap was used for the cellphone surface. Totally, 400 samples were assessed by swab for the presence of HA-MRSA in this study. The samples were cultivated at nutrient broth medium at $37^{\circ} \mathrm{C}$ for $24 \mathrm{~h}$; then recultivated on the agar medium. After $18 \mathrm{~h}$ of incubation, the colonies were stained by gram method. Gram-positive cocci were detected, and Staphylococcus aureus was evaluated by biochemical tests such as catalase, coagulase, Mannitol, DNase and Novobiocin Test. Also, the Nitrocefin test was used for identifying the beta-lactamase positive Staphylococcus. ${ }^{11}$ To identify Staphylococcus aureus resistance to methicillin (MRSA), the concentration equivalent to 0.5 McFarland standard was used to cultivate on Müller Hinton agar. The Kirby-Bauer method test was performed for antibiotics including ceftriaxone, vancomycin, piperacillin, imipenem, cefoxitin, cefotaxime. ${ }^{12}$

\section{Results}

After conducting biochemical tests, 251 positive samples of Staphylococcus aureus were detected; including 164 samples from nasal secretions and 87 from mobile phones. Data is provided in detail in table number 1 . The rate of positive beta-lactamase staphylococci isolated from the nose and the cell phone was $31 \%$ (62) and $12 \%{ }^{24}$ among the staff of the hospital, respectively (see Table 1).

86 samples of beta-lactamase positive Staphylococcus aureus were evaluated from the point of the sensitivity test. 17 were resistant to cefoxitin, piperacillin, ceftriaxone, and cefotaxime; interestingly, one of these 17 samples showed resistance to vancomycin (Table 2). Also. In this study, 24 samples from the cell phone of the Emergency staff and their nasal swab was similar for the type of HA-MRSA bacteria and also for the antibiotic susceptibility. The surface of the mobile phone and nasal discharge of $12 \%$ of the studied personnel were infected with HAMRSA. It is possible that the bacteria transmitted from the nasal discharge to the cellular phone surface, which leads to cell phone contamination. In this study, the prevalence of beta-lactamase positive Staphylococcus aureus (isolated from nasal discharge) was $31 \%$ in Ghaem Hospital emergency department staff.
Table 1

Frequency of $\beta$-lactamase positive Staphylococcus aureus from mobile samples and nasal secretions.

\begin{tabular}{lll}
\hline & Number & beta-lactamase Positive \\
\hline Mobile surface samples & 200 & $24(12 \%)$ \\
Nasal discharge samples & 200 & $62(31 \%)$ \\
\hline
\end{tabular}

\section{Discussion}

Increasing antibiotic-resistant microorganisms is one of the most serious problems in health care systems. ${ }^{13-18}$ One of the most common antibiotic-resistant bacteria is MRSA. Identification of MRSA contaminated staff in hospital wards can prevent the spread of this type of resistance. ${ }^{10}$ Our result showed that the prevalence of MRSA isolated from the nose and the cell phone was $31 \%$ (62) and $12 \%^{24}$ among the staff of the hospital, respectively.

Rahimi et al. reported the rate of isolated MRSA from different hospitals in Tehran; it was $29.7 \%$, which was almost consistent with our results. $^{3}$ The study by Kannan Sridhara et al., in 2014 reported 58.8\% positive MRSA among nurses in a tertiary care hospital. ${ }^{19}$ In the study by Nabil Abdullah et al. in Shifa hospital, MRSA carriage rate was highest among nurses. ${ }^{20}$ The Study by Jann-Tay Wang et al. among adults in Community Settings in Taiwan indicated that the MRSA colonization rate was $3.8 \%$. Of course, it is necessary to mention this point that the samples were collected from the community, not healthcare personnel. ${ }^{21}$ In a study done by Schultz, $7.6 \%$ of the isolates from the nose of training soldiers were identified as MRSA; all of them were sensitive to vancomycin 22 . Another study on the military population and their families in the United States by Kenner et al., in 2003 showed that $40 \%$ of the people had nasal staphylococci and $2 \%{ }^{8}$ carried MRSA in the nose. ${ }^{23}$

The study by Solmaz Dibah, which was done in Iran in 2014, found MRSA in $46.3 \%$ of isolates. Most of the MRSA strains (68.4\%) were obtained from ICU hospitalized patients; all were susceptible to vancomycin24. The study by Ramin Dibaj et al. in Esfahan Kindergartens demonstrated that $9.5 \%$ of children carried MRSA. All MRSA were susceptible to vancomycin 25 .

In some studies, the prevalence of MRSA contamination in mobile phones was investigated. For example, a study done by Selim et al. in Egypt, for the identification of microbial contamination of mobile phones in a health care setting. The most prevalent bacterial contaminants were methicillin-resistant S. aureus $(53 \%){ }^{26}$ Tambe et al. reported $56.16 \%$ as the rate of contamination of cell phones among Health Care personnel. ${ }^{27}$ In the study by Shekhar Pal et al. bacterial growth was shown in $81.8 \%$ of mobile phone swab samples. The most predominant isolates were Staphylococcus aureus which can transfer to the patients. ${ }^{28}$ In the study done by Almugadam BS et al., the prevalence of positive culture among mobile phone, ear, and hand swabs were $95 \%$ (57/60), 100\% (30/30) and 96.7\% (29/30), respectively; the frequency of MRSA was $98.6 \% .^{29}$

In a study for the determination of antibiotic resistance of $S$. aureus isolated from door handles and other points of contact in public hospitals in Ghana, $17 \%$ were putative MRSA isolates. Also, One MRSA isolate was resistant to all the used antibiotics (cefoxitin, oxacillin, ciprofloxacin, erythromycin, tetracycline, ampicillin, streptomycin, and sulfamethoxazole-trimethoprim. ${ }^{21}$

Investigation of Prevalence and antibiotic resistance pattern of methicillin-resistant Staphylococcus aureus in the Orthopaedic Hospital in Nigeria by Udobi et al. showed resistance pattern of: ampicillin $100 \%$, pefloxacin $90.9 \%$, ceftriaxone $69.7 \%$, gentamicin $54.5 \%$, and ciprofloxacin $51.5 \%{ }^{30}$ The study by Anupriya et al. revealed that microbial contamination of mobile phones in a tertiary care hospital was 87.3\% and 56.4\% among health care workers versus non-health care workers, but there was no MRSA. ${ }^{31}$ A Study by Nirmal et al. among 
Table 2

Antibiogram results of Staphylococcus aureus isolated from nasal secretion and phone surfaces.

\begin{tabular}{|c|c|c|c|c|c|c|c|}
\hline \multirow[t]{2}{*}{ Bacteria } & \multicolumn{3}{|c|}{ Nasal secretion } & \multicolumn{4}{|l|}{ Phone surface } \\
\hline & Antibiotic & number & $\begin{array}{l}\text { Sensitive } \\
\%))\end{array}$ & Resistant (\%) & number & sensitive (\%) & Resistant (\%) \\
\hline S. aureus & vancomycin & 62 & $61(98.84 \%)$ & $1(1.16 \%)$ & 24 & $24(100 \%)$ & $100 \%$ \\
\hline S. aureus & ceftriaxone & 62 & $62(100 \%)$ & $100 \%$ & 24 & $20(83.3 \%)$ & $4(16.6 \%)$ \\
\hline S. aureus & cefotaxime & 62 & $62(100 \%)$ & $100 \%$ & 24 & $20(83.3 \%)$ & $4(16.6 \%)$ \\
\hline S. aureus & cefoxitin & 62 & $60(96.7 \%)$ & $2(3.3 \%)$ & 24 & $24(100 \%)$ & $100 \%$ \\
\hline S. aureus & piperacillin & 62 & $62(100 \%)$ & $100 \%$ & 2 & $22(91.6 \%)$ & $2(8.4 \%)$ \\
\hline S. aureus & imipenem & 62 & $58(93.54 \%)$ & $4(6.46 \%)$ & 0 & $24(100 \%)$ & $24(100 \%)$ \\
\hline S. aureus & aztreonam & 62 & $58(93.54 \%)$ & $4(6.46 \%)$ & 0 & $24(100 \%)$ & $24(100 \%)$ \\
\hline
\end{tabular}

health-care workers, explained that HA-MRSA carriage was seen in 47 individuals in their nasal vestibules; the colonization rate was $15.1 \%$. HA-MRSA was also isolated from the cell phone surfaces.

In this study, antibiotic susceptibility tests revealed that the HAMRSA isolates are sensitive to most antibiotics, but there was a high rate of resistance against erythromycin (48\%) and clindamycin $(29 \%){ }^{32}$ In contrast, our current study shows a significantly higher percentage of nasal carriage of HA-MRSA. However, our results are similar to the Chinese study conducted in $2011 .^{33}$ The prevalence of MRSA indicates that the beta-lactamase positive staphylococci in nonhospital place are much lower in comparison to hospital, which can be a hazard to hospital staff.

\section{Conclusion}

Our result showed that the prevalence of MRSA is lower in nonhospital place in comparison to the hospital, and also since the cell phone is used in different places, can easily transmit the bacteria from the hospital environment to children, older people and who have a weakened immune system. Therefore, it is suggested to evaluate all people working in hospitals regarding the contamination to MRSA. Meanwhile, cell phone usage should be prohibited in the hospital sections. Disinfection of the mobile phone when leaving the hospital along with hand, face, and nose washing should be taken seriously.

\section{Declaration of competing interest}

The authors whose names are listed immediately below certify that they have NO affiliations with or involvement in any organization or entity with any financial interest (such as honoraria; educational grants; participation in speakers' bureaus; membership, employment, consultancies, stock ownership, or other equity interest; and expert testimony or patent-licensing arrangements), or non-financial interest (such as personal or professional relationships, affiliations, knowledge or beliefs) in the subject matter or materials discussed in this manuscript.

\section{References}

1. Ramana KV, Mohanty SK, Wilson CG. Staphylococcus aureus colonization of anterior nares of school going children. Indian J Pediatr. 2009;76(8) 813-6.

2. Klein E, Smith DL, Laxminarayan R. Community-associated methicillin-resistant Staphylococcus aureus in outpatients, United States, 1999-2006. Emerg Infect Dis. 2009;15(12):1925.

3. Rahimi F, Karimi S. Antibiotic resistance pattern and prophage typing of methicillin resistant Staphylococcus aureus strains isolated from chicken husbandries in tehran. Iran. J. Clin. Infect. Dis. Trop. Med. 2014;18(62):17-22.

4. Plata K, Rosato AE, Wegrzyn G. Staphylococcus aureus as an infectious agent: overview of biochemistry and molecular genetics of its pathogenicity. Acta Biochim Pol. 2009;56(4):597.

5. Havaei SA, Vidovic S, Tahmineh N, et al. Epidemic methicillin-susceptible Staphylococcus aureus lineages are the main cause of infections at an Iranian university hospital. J Clin Microbiol. 2011;49(11):3990-3993.

6. Havaei SA, Halaji M, Vidovic S, et al. Prevalence and genotyping of methicillin- resistant and-susceptible Staphylococcus aureus strains isolated from patients in a university hospital, Isfahan, Iran. Jundishapur J Microbiol. 2017;10(5).

7. Rezazadeh M, Yousefi MR, Sarmadian H, Ghaznavirad E. Antibiotic Profile of Methicillin-Resistant Staphylococcus aureus with Multiple-Drug Resistances Isolated from Nosocomial Infections in Vali-Asr Hospital of Arak. 2013; 2013.

8. Sobhani Poor MH, Mansouri S, Saeidadeli N. Prevalence of methicillin-resistant Staphylococcus aureus (MRSA) and antibiotic resistance patterns of the isolates from the nose of training soldiers in kerman in 2012. Iran J Med Microbiol 2014;8(3):15-21

9. Otter JA, French GL. Molecular epidemiology of community-associated meticillinresistant Staphylococcus aureus in Europe. Lancet Infect Dis. 2010;10(4):227-239.

10. Feßler AT, Schünemann R, Kadlec K, et al. Methicillin-resistant Staphylococcus aureus (MRSA) and methicillin-resistant Staphylococcus pseudintermedius (MRSP) among employees and in the environment of a small animal hospital. Vet Microbiol. 2018;221:153-158.

11. Safdari H, Neshani A, Sadeghian A, Ebrahimi M, Iranshahi M, Sadeghian H. Potent and selective inhibitors of class A $\beta$-lactamase: 7-prenyloxy coumarins. $J$ Antibiot. 2014;67(5):373.

12. Hudzicki J. Kirby-Bauer Disk Diffusion Susceptibility Test Protocol. 2009; 2009.

13. Appelbaum PC. Microbiology of antibiotic resistance in Staphylococcus aureus. Clin Infect Dis. 2007;45(Supplement_3):S165-S170.

14. Khaledi A, Esmaeili D, Jamehdar SA, Esmaeili S-A, Neshani A, Bahador A. Expression of MFS efflux pumps among multidrug resistant Acinetobacter baumannii clinical isolates. Der Pharm Lett. 2016;8:262-267.

15. Esmaeili D, Daymad SF, Neshani A, Rashki S, Marzhoseyni Z, Khaledi A. Alerting prevalence of MBLs producing Pseudomonas aeruginosa isolates. Gene Reports. 2019;16:100460

16. Kholoujini M, Karami P, Khaledi A, Neshani A, Matin P, Alikhani MY. Identification of Pathogenic Bacteria in Blood Cultures and Susceptibility Testing of Isolates with Various Antibiotics. 2016; 2016

17. Neshani A, Zare H, Eidgahi MRA, Khaledi A, Ghazvini K. Epinecidin-1, a highly potent marine antimicrobial peptide with anticancer and immunomodulatory activities. BMC Pharmacol. Toxicol. 2019;20(1):33.

18. Mansury D, Khaledi A, Ghazvini K, et al. Study of bacterial infections among patients receiving kidney transplant in Mashhad, Iran. Exp. Clin. Transplant. 2018;16(3):282-286.

19. Sridharan K, Mallik A, Madan M. Prevalence of methicillin-resistant Staphylococcus aureus among hospital healthcare workers in a tertiary care hospital: a cross-sectional study. Int. J. Health. Allied Sci. 2016;5(3):169.

20. El Aila NA, Al Laham NA, Ayesh BM. Nasal carriage of methicillin resistant Staphylococcus aureus among health care workers at Al Shifa hospital in Gaza Strip. BMC Infect Dis. 2017;17(1):28.

21. Saba CKS, Amenyona JK, Kpordze SW. Prevalence and pattern of antibiotic resistance of Staphylococcus aureus isolated from door handles and other points of contact in public hospitals in Ghana. Antimicrob Resist Infect Contr. 2017;6(1):44.

22. Schultz M. Methicillin-resistant Staphylococcus aureus (MRSA) what the pharmacist should know. SA Pharm J. 2009;76(5):28-30.

23. Kenner J, O'connor T, Piantanida N, et al. Rates of carriage of methicillin-resistant and methicillin-susceptible Staphylococcus aureus in an outpatient population. Infect Contr Hosp Epidemiol. 2003;24(6):439-444.

24. Dibah S, Arzanlou M, Jannati E, Shapouri R. Prevalence and antimicrobial resistance pattern of methicillin resistant Staphylococcus aureus (MRSA) strains isolated from clinical specimens in Ardabil, Iran. Iran J Microbiol. 2014;6(3):163.

25. Dibaj R, Shoaei P, Shojaei H. Study of prevalence and characteristics of Staphylococcus aureus and CA-MRSA nasal colonization in 2-5 Years old children in isfahan. Iran J Med Microbiol. 2014;8(3):22-30.

26. Selim HS, Abaza AF. Microbial Contamination of Mobile Phones in a Health Care Setting in Alexandria, Egypt. vol. 10. GMS hygiene and infection control; 2015.

27. Tambe NN, Pai C. A study of microbial flora and MRSA harboured by mobile phones of health care personnel. Int J Recent Trends Sci Technol. 2012;4:14-18.

28. Pal S, Juyal D, Adekhandi S, et al. Mobile phones: reservoirs for the transmission of nosocomial pathogens. Adv Biomed Res. 2015;4.

29. Almugadam BSAH, Osman MB, Omer SM. Frequency of MRSA isolates in mobile phones, ears and hands of healthcare workers. Antimicrob Agents. 2018;4(1):161.

30. Udobi C, Obajuluwa A, Onaolapo J. Prevalence and antibiotic resistance pattern of methicillin-resistant Staphylococcus aureus from an orthopaedic hospital in Nigeria. 
BioMed Res Int. 2013;2013.

31. Anupriya A, Puhalenthi K, Prethi R, Hemasri V. Microbial contamination of mobile phones in a teritary care hospital. Int. J. Community Med. Public Health. 2018;5(6):2313-2316.

32. Pathare NA, Asogan H, Tejani S, et al. Prevalence of methicillin resistant
Staphylococcus aureus [MRSA] colonization or carriage among health-care workers. J. Infect. Public Health. 2016;9(5):571-576.

33. Song J-H, Hsueh P-R, Chung DR, et al. Spread of methicillin-resistant Staphylococcus aureus between the community and the hospitals in Asian countries: an ANSORP study. J Antimicrob Chemother. 2011;66(5):1061-1069. 